\title{
Leucaemia cutis bei akuter myeloischer Leukämie
}

\section{Leukemia cutis in a Patient with Myelogenous Leukemia}

Autoren

Institut
G. Wagner, M. M. Sachse

Hautklinik, Klinikum Bremerhaven Reinkenheide (Chefarzt: Dr. G. Wagner)
Bibliografie

DOI http://dx.doi.org/ $10.1055 / \mathrm{s}-0030-1256100$ Akt Dermatol 2011; 37: 91-95 () Georg Thieme Verlag KG Stuttgart · New York ISSN 0340-2541

\section{Korrespondenzadresse} Dr. Gunnar Wagner Klinikum Bremerhaven Reinkenheide Postbrookstr. 103 27574 Bremerhaven Gunnar.Wagner@ klinikum-bremerhaven.de

\section{Zusammenfassung $\nabla$}

Ohne Einschränkung des Allgemeinbefindens entwickelten sich bei einem 63-jährigen Patienten purpuriforme Papeln und Plaques am Stamm und proximal an den Extremitäten. Die histopathologischen und hämatoonkologischen Untersuchungen führten zur Diagnose einer Leucaemia cutis bei akuter myeloischer Leukämie vom myelomonozytären Subtyp. Die Leucaemia cutis wird als primäre Manifestation einer systemischen

\section{Einleitung}

\section{$\nabla$}

Die klinische Symptomatik der Leukämien wird im Wesentlichen durch ihren biologischen Verlauf geprägt. Dabei kommt es nach Ausbreitung der malignen Zellreihen im Knochenmark und deren Ausschwemmung in das periphere Blut zu einem Befall extramedullärer Organe. Auf der Grundlage der Dynamik ihres spontanen, nicht durch eine Behandlung modifizierten Verlaufes und anhand des Differenzierungsgrades der neoplastischen Zellen sind die Leukämien in akute und chronische sowie in lymphatische und myeloische Leukämien eingeteilt worden. Die Subtypisierung erfolgte auf Empfehlung der FrenchAmerican-British Cooperative Group (FAB), wobei die akute myeloische Leukämie in die Typen M0M7 differenziert wurde. In einer 2001 veröffentlichten und vor Kurzem modifizierten WHO-Klassifikation wurden erstmalig auch genetische Veränderungen bei den verschiedenen Formen berücksichtigt [1,2]. Bei den akuten Leukämien ist der meist dramatische Krankheitsverlauf auf die schnell zunehmende Knochenmarkinsuffizienz zurückzuführen, die mit septischen Temperaturen, Infektionen und Blutungen einhergeht. Die chronischen Leukämieformen stellen hingegen häufiger Zufallsbefunde dar, z. B. bei routinemäßigen Blutbildbestimmungen, oder sind von einer
Leukämie in etwa 7\% der Fälle beobachtet. Das klinische Bild der Leucaemia cutis ist durch eine unterschiedliche Morphologie der Befunde gekennzeichnet. $\mathrm{Zu}$ den Primäreffloreszenzen zählen Erytheme, Papeln, Knoten, Blasen und Ulzera. Entsprechend umfangreich sind die zu berücksichtigenden Differenzialdiagnosen, zu denen unter anderem Arzneimittelexantheme, Vaskulitiden und Ulzera unterschiedlicher Genese gezählt werden müssen.

uncharakteristischen Klinik begleitet, von subfebrilen Temperaturen, Gewichtsverlust oder allgemeinem Unwohlsein $[3,4]$. In der dermatologischen Praxis ergibt sich die klinische Verdachtsdiagnose einer Leucaemia cutis insbesondere bei den akuten Leukämieformen aus dem Zusammenspiel der allgemeinen Krankheitssymptomatik und den dermatologischen Befunden. Dass die für die Diagnose einer Leucaemia cutis häufig richtungweisende Allgemeinsymptomatik im Einzelfall auch fehlen kann, belegt die hier vorgestellte Kasuistik.

\section{Kasuistik \\ $\nabla$}

\section{Anamnese}

Eine Woche vor stationärer Aufnahme hatte der 63-jährige Patient erstmals juckende Hautveränderungen am Stamm bemerkt. Bei Zunahme der Befunde erfolgte die Klinikeinweisung durch eine niedergelassene Dermatologin unter der Diagnose einer Vaskulitis. Infekte der oberen Luftwege, Diarrhoen oder Einschränkungen des Allgemeinbefindens ließen sich nicht erfragen. Die Einnahme von Medikamenten verneinte der Patient. 


\section{Klinischer Befund}

In disseminierter Aussaat zeigten sich am Stamm und proximal an den Extremitäten maximal fingernagelgroße, zusammengesetzt aufgebaute Läsionen, bestehend aus purpuriformen Papeln und Plaques im Zentrum und einem unscharf begrenzten Erythem im Randbereich ( $\bullet$ Abb. 1 u. 2). Auffällig derbe, plattenartige Resistenzen in der Tiefe tastbar. Im Bereich der Mundschleimhaut einzelne linsengroße Petechien. Der übrige körperliche Untersuchungsbefund war unauffällig, insbesondere keine peripheren Lymphknoten und keine Splenomegalie tastbar.

\section{Befunde diagnostischer Untersuchungen \\ Histopathologische Befunde}

Biopsie Oberarm rechts: Bei fehlendem Epidermotropismus zeigen sich überwiegend perivaskuläre, knotig akzentuierte Infiltrate im oberen und mittleren Korium, bestehend aus kleinen bis mittelgroßen Zellen, vermutlich histiozytärer Herkunft. Keine Mitosefiguren nachweisbar ( $\bullet$ Abb. 3). Immunhistochemisch exprimieren die atypischen Zellen CD4, CD68 und Lysozym. Kein Nachweis von CD5, CD20, CD23, CD45RO (UCHL1), CD 56 und CD117; Myeloperoxidase negativ.

Befundinterpretation: V.a. Infiltrat einer Leukämie, am ehesten myeloider Herkunft, allerdings mit fehlender Immunexpression für typische Marker der myeloischen Leukämie (Dr. C. Diaz, Dermatopathologie Freiburg).

Knochenmarkbiopsie Beckenkamm: Mittelbreite Spongiosabälkchen. Im polarisierten Licht unauffällige Lamellierung der Knochenbälkchen. Zellularität erhöht, Fettgehalt um 5\%. Zahlreiche Megakaryozyten mit deutlicher morphologischer Atypie. Granulozytopoese mit Reifungsstörung bei Verminderung der ausgereiften Form. Massive Vermehrung überwiegend Chloracetatesterase-negativer Blasten. Verminderte Erythrozytopoese. Immunhistochemisch erweisen sich die Blasten positiv für CD34, CD117 und überwiegend auch für Myeloperoxidase.

Diagnose: Akute myelomonozytäre Leukämie M4 nach FAB (Dr. M. Tiemann, Dr. H. Qiu, Institut für Hämatopathologie Hamburg).

\section{Laborbefunde}

Blutbild: Leukozyten 63,6 (NW: 4,0-10,5/nl), Erythrozyten 3,3 (NW: 3,5-5,8/pl), Hämoglobin 9,9 (NW: 14,0-18,0 g/dl), Hämatokrit 31,7 (NW: 38-51\%), MCV 95,9 (NW: 83-88fl), RDW 16,6 (NW: $11-16 \% \mathrm{VC}$ ), Thrombozyten 25 (NW: 130-450/nl)

Differenzialblutbild: Stabkernige 1 (NW: <6\%), Segmentkernige 12 (NW: $45-85 \%$ ), Basophile 2 (NW: 0-3\%), Monozyten 17 (NW: $1-11 \%$ ), Lymphozyten 9 (NW: $10-50 \%$ ), atypische Zellen 59 (NW: $0-3 \%$ ).

BSG 61/94 (NW: 10-20 mm/h). Nierenpflichtige Substanzen, Lebertransaminasen, Bilirubin, Eiweißelektrophorese und Urinstatus ohne pathologische Befunde.

\section{Ergänzende Untersuchungen}

In der Oberbauchsonografie zeigte sich eine Splenomegalie von $16,3 \mathrm{~cm}$. Rö-Thorax, CT-Thorax, Skelettszintigraphie sowie Lymphknoten-Sonographien der Achseln und Leisten ergaben keine richtungweisenden pathologischen Befunde.

\section{Therapie und Verlauf}

$\nabla$

Aufgrund der hämatologischen Befunde wurde der Patient umgehend in eine onkologische Klinik verlegt. Nach endgültiger Sicherung der Diagnose einer akuten myeloischen Leukämie

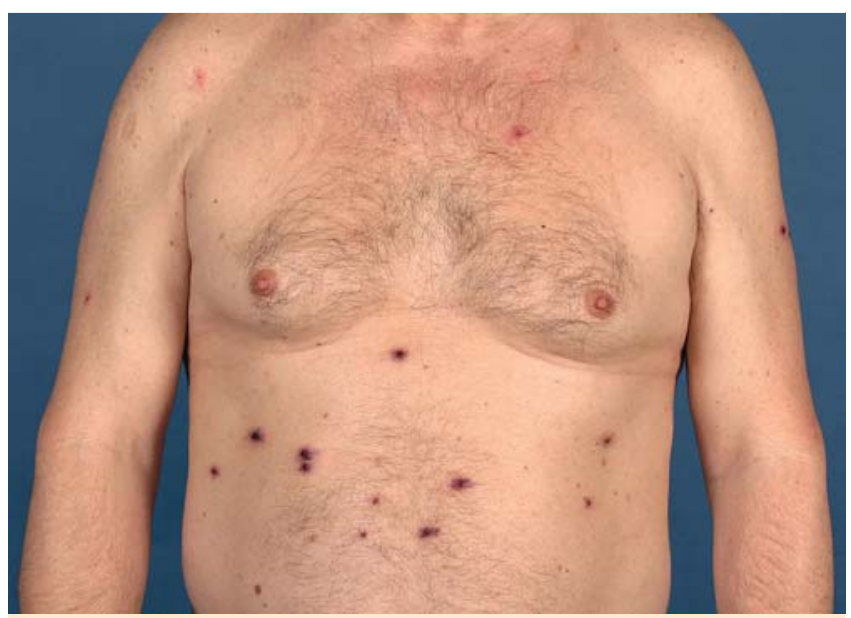

Abb. 1 Leucaemia cutis mit disseminierter Verteilung der Läsionen am Stamm und den Oberarmen.

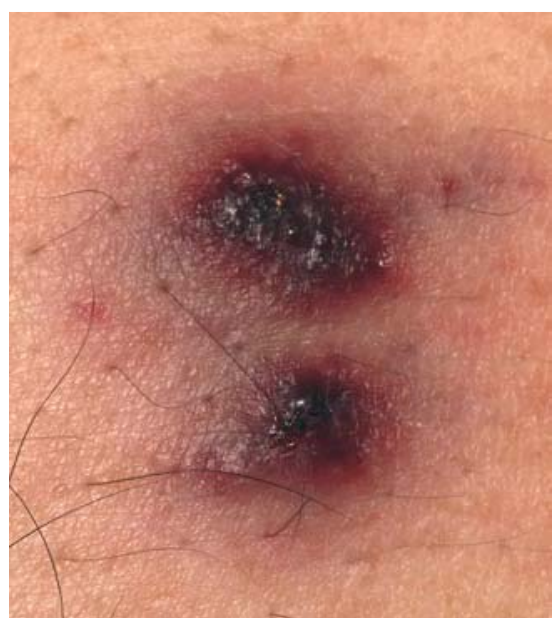

Abb. 2 Detailaufnahme: zwei konfluierende, unscharf begrenzte Erytheme mit infiltrierten, hämorrhagischen Zentren.

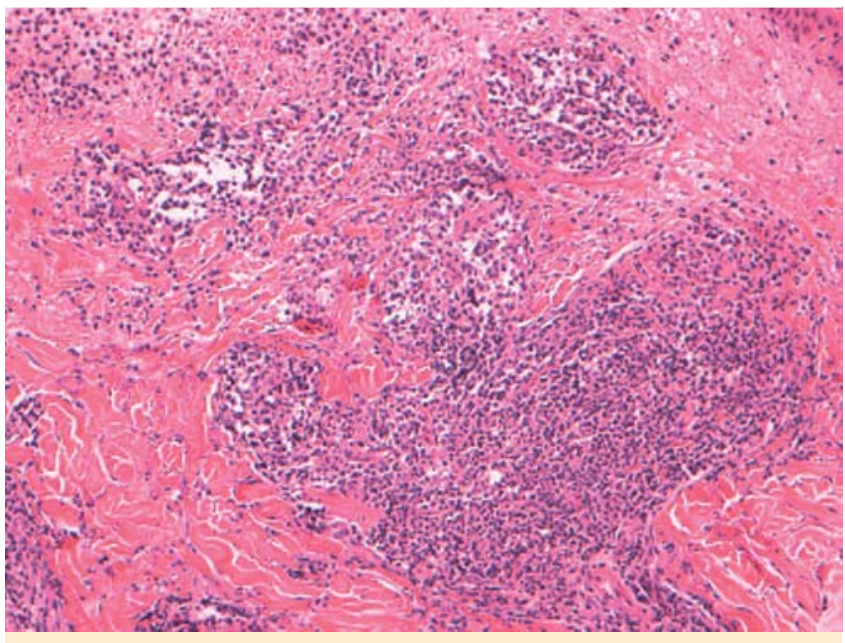

Abb. 3 Perivaskuläre, knotig akzentuierte Infiltrate atypischer Zellen im oberen und mittleren Korium (HE 1:200).

vom myelomonozytären Subtyp erfolgten Induktionschemotherapien nach dem DA-Schema (Daunorubicin, Ara-C) und dem HAM-Protokoll (Ara-C, Mitoxantron). Beide Behandlungsverfahren führten zu keiner Remission. Auch eine wesentliche Rückbildung der dermatologischen Befunde konnte nicht beobachtet werden. Vor Durchführung einer geplanten Stammzelltransplantation verstarb der Patient drei Monate nach Erstdiagnose. 


\section{Diskussion}

\section{V}

Die dermatologischen Manifestationen der Leukämien werden in der Praxis auch heute noch auf der Grundlage historischer Befunde in spezifische und unspezifische Hautveränderungen eingeteilt. Die spezifischen zeichnen sich durch kutane Infiltrationen leukämischer Zellen aus und werden unabhängig von der jeweils vorliegenden klinischen Morphologie als Leucaemia cutis bezeichnet. Die unspezifischen Hautveränderungen hingegen sind auf einen Funktionsverlust der Leukozyten zurückzuführen, Folge der entstehenden Knochenmarkinsuffizienz oder Ausdruck einer kutanen Paraneoplasie [5,6]. Diese Klassifikation spezifischer und unspezifischer Hautveränderungen beruht auf dem Nachweis leukämischer Zellen, ein Nachweis, der jedoch abhängig ist von den ursprünglich vorhandenen histopathologischen Untersuchungsmethoden. Mit den heute zur Verfügung stehenden immunhistochemischen und molekulargenetischen Techniken lassen sich leukämische Zellen auch in den unspezifischen Hautveränderungen der Leukämien und in anderen, unabhängig von einer bestehenden Leukämie vorhandenen Dermatosen detektieren, z.B. in Karzinomen, Herpes-Infektionen, Ekzemen oder der Psoriasis vulgaris [7-11]. Das Auftreten von Leukämiezellen in kutanen Strukturen ist somit als alleiniges Kriterium für die Diagnose einer Leucaemia cutis nicht mehr ausreichend. Demzufolge erscheint es notwendig, die ursprüngliche Definition der Leucaemia cutis zu modifizieren [10]. Neben dem Nachweis leukämischer Infiltrate sollten dabei unserer Meinung nach nur die in ihrer Morphologie zwar variablen, insgesamt aber doch typischen klinischen Läsionen der Leucaemia cutis berücksichtigt werden bei gleichzeitiger Abgrenzung eigenständiger Dermatosen anderer Ätiologie. Die Leucaemia cutis kann mit unterschiedlichen Häufigkeiten bei allen Leukämieformen beobachtet werden. Die Einteilung der Leukämien erfolgt anhand der Empfehlung der French-American-British Cooperative Group (FAB), wobei die akute myeloische Leukämie in die Subtypen M0 - M7 differenziert werden kann. Diese Klassifikation wurde 2001 durch die WHO um molekulargenetische Charakteristika erweitert $[1,2]$. Bei der chronisch lymphatischen Leukämie, der in den westlichen Industrienationen häufigsten Leukämieform, betrug der Anteil von spezifischen Hautveränderungen betroffener Patienten in mehreren Untersuchungen 8-27\% [12,13]. In der Gruppe der akuten myeloischen Leukämie mit ihren zahlreichen Subtypen wurden in 2-13\% der Fälle eine Leucaemia cutis nachgewiesen, mit 25-33\% am häufigsten bei der akuten Monozytenleukämie [14-17]. Deutlich seltener manifestieren sich spezifische Hautveränderungen bei der akuten lymphatischen und der chronisch myeloischen Leukämie. Die Häufigkeit der Leucaemia cutis wird bei diesen Leukämieformen mit 2-3\% angegeben $[15,18]$. Für die Dermatologen von besonderem Interesse ist der zeitliche Zusammenhang zwischen dem Auftreten einer Leucaemia cutis und dem Zeitpunkt der Leukämiediagnose. In einer Studie von Su et al., bei der Daten von 42 Patienten ausgewertet wurden, war die Leucaemia cutis bei 55\% der Fälle im Verlauf einer bereits bekannten Leukämie aufgetreten, während bei $38 \%$ der Betroffenen beide Diagnosen gleichzeitig gestellt worden waren. Bei 7\% der Patienten zeigte sich die Leucaemia cutis als Initialsymptom einer Leukämie. Darüber hinaus können die Hautveränderungen in Einzelfällen als so genannte aleukämische Leucaemia cutis den spezifischen hämatoonkologischen Befunden um Wochen oder Monate vorausgehen, was die Bedeutung der dermatologischen Diagnose für das Erkennen und die rechtzeitige Therapie der Leukämien unterstreicht [19]. Die klinische Mor- phologie der Leucaemia cutis zeichnet sich durch verschiedene Primäreffloreszenzen und deren Verteilung aus. Die häufigsten Läsionen der Leucaemia cutis sind Papeln und Knoten. In einzelnen Untersuchungen wurde ihr Anteil mit bis zu $60 \%$ angegeben. Während die Papeln mit einem Durchmesser von wenigen Millimetern häufig in exanthematischer oder umschrieben gruppierter Aussaat vorliegen und Hunderte von Exemplaren umfassen können, finden sich die typischerweise $2-3 \mathrm{~cm}$ durchmessenden Knoten in einer disseminierten Verteilung weniger oder einiger Dutzend Exemplare. Die Farbtöne der Papeln und Knoten variieren zwischen hell- bis dunkelrot, rotbraun, violett und hämorrhagisch. Der Palpationsbefund der Morphen ist weich oder fest, jedoch typischerweise nicht hart [5,19-26]. Die Lokalisation der Morphen ist durch den Systemcharakter der Leukämien geprägt, sodass die generalisierte Manifestation der Leucaemia cutis als wichtiger Befund angesehen werden kann. Bei einem multiplen Auftreten der Läsionen, dem Regelfall der Leucaemia cutis, sind am Stamm der Rücken und die Thoraxorgane sowie darüber hinaus die Arme und Beine am häufigsten betroffen. Aber auch bei der Stamm- und Extremitäten-betonten Manifestation der Leucaemia cutis können weitere Läsionen zusätzlich am Kopf, am Nacken und palmoplantar vorhanden sein. Im Gegensatz zum multiplen Charakter der Papeln und Knoten müssen einzelne, maximal faustgroße, oberflächlich auch ulzerierende Tumoren als selten angesehen werden [5,14,27]. Neben den papulösen und knotigen Läsionen finden sich als weitere klinisch-morphologische Varianten der Leucaemia cutis Erytheme und erythematöse Plaques, die bei etwa 20 - 25\% der Patienten beobachtet werden. Die unscharf begrenzten, münz- bis handtellergroßen Erytheme können konfluieren, weisen rote bis rotbraune, aber auch blaugraue Farbtöne auf und sind bisweilen druckdolent $[9,20,28]$. Die Oberfläche der Erytheme kann durch eine ausgeprägte pityriasiforme Schuppung gekennzeichnet sein oder Erosionen mit Krusten aufweisen [8]. Bei Zunahme der zunächst nur tastbaren Infiltrationen der Erytheme entstehen plattenartige, flach erhabene Plaques von jetzt meist derber Konsistenz $[5,6,29]$. Auch die Erytheme und Plaques können in allen Lokalisationen am Integument auftreten, möglicherweise etwas häufiger an den Unterschenkeln und im Gesicht, was für die zu erwägenden klinischen Differenzialdiagnosen von Bedeutung ist [5]. Als seltene Manifestationen der Leucaemia cutis gelten Ulzera und Blasen. Die mit einem Durchmesser von meist nur wenigen Zentimetern typischerweise kleinen Ulzera treten überwiegend singulär und nur vereinzelt in Mehrzahl auf [30-32]. Der Ulkusgrund kann durch festsitzende, purulente Beläge gekennzeichnet sein, der Rand erscheint bisweilen wallartig aufgeworfen [31]. Die Ulzera können sich in ungewöhnlichen Lokalisationen entwickeln, z. B. skrotal, inguinal oder im Bereich des Gesichtes [30,31]. Die ungewöhnlichste klinische Manifestationsform leukämischer Infiltrate stellen zweifellos Blasen dar. An den Streckseiten der Finger, aber auch disseminiert am Stamm werden bis zu münzgroße, auf rotem Grund stehende, teilweise hämorrhagisch eingetrübte Blasen beschrieben $[6,8,33]$. Als histopathologisches Korrelat der bullösen Leucaemia cutis wurde vereinzelt eine leukämische Vaskulitis nachgewiesen [33]. Neben der Manifestation am Integument kann die Leucaemia cutis auch im Bereich der Schleimhäute auftreten. Hier gilt die Gingivahyperplasie mit ihrer ausgeprägten Neigung zu hämorrhagisch-nekrotischen Ulzerationen als typisches Beispiel für eine leukämische Infiltration der Mundschleimhäute [25]. Die beschriebenen Morphen, insbesondere die makulösen Läsionen, treten häufig kombiniert auf. Eine spezielle Zuordnung der dermatologischen Befunde zu 
einzelnen Leukämieformen ist, von Ausnahmen abgesehen, zu denen z.B. die Gingivahyperplasie bei der akuten myeloischen Leukämie gezählt wird, nicht möglich.

Die klinischen Differenzialdiagnosen sind zahlreich und richten sich im Einzelfall nach der vorliegenden Morphologie der Leucaemia cutis. Bei exanthematisch auftretenden Papeln ist an eine kleinknotige Sarkoidose oder an papulöse Arzneimittelexantheme zu denken, während man bei einer umschriebenen Manifestation der papulösen Läsionen Speicherdermatosen berücksichtigen muss, z.B. den Lichen amyloidosus oder die Mucinosis papulosa $[19,20,34]$. Disseminierte oder einzeln stehende Knoten gilt es von kutanen Lymphomen oder Metastasen viszeraler Malignome abzugrenzen [14,27]. In Abhängigkeit von der Lokalisation können Erytheme der Leucaemia cutis an den Unterschenkeln mit Ekzemen oder bei zusätzlich vorhandener Druckdolenz auch mit einem Erythema nodosum verwechselt werden. Schuppende Erytheme im Gesicht imitieren bisweilen einen Lupus erythematodes oder bei deutlicher Infiltration einen Morbus Morbihan $[5,35,36]$. Generalisierte Erytheme mit Schuppung, Lichenifikationen und Vesikeln entsprechen dem Bild einer ekzematoiden Erythrodermie [8]. Infiltrierte Plaques der Leucaemia cutis lassen sich klinisch nicht von einer Mycosis fungoides unterscheiden [29]. Alle makulo-papulösen oder plaqueförmigen Läsionen der Leucaemia cutis mit einer hämorrhagischen oder purpuriformen Komponente können verschiedene Formen einer Vaskulitis nachahmen, wie dies auch bei dem hier vorgestellten Patienten der Fall gewesen ist [30,37,38]. Die Differenzialdiagnosen der Ulzera umfassen bei der Leucaemia cutis das Ulcus cruris venosum, ulzerierte kutane Malignome und das Pyoderma gangraenosum, das auch als unspezifische Hautveränderung bei Leukämien vorkommen kann [5,31]. Die klinische Morphologie der bullösen Leucaemia cutis lässt sich palmoplantar mit den Befunden eines Erythema exsudativum multiforme und der bullösen Kontaktdermatitis vergleichen oder entspricht an den Extremitäten und am Stamm bei größeren blasigen oder postbullösen Läsionen den typischen Befunden der bullösen Autoimmundermatosen $[6,8,33]$.

Die Vielzahl der genannten Differenzialdiagnosen und die Erkenntnis, dass eine Leucaemia cutis als Erstsymptom einer Leukämie auftreten kann, unterstreicht im Übrigen auch wieder einmal nachdrücklich die Bedeutung der Dermatologie für die Diagnose internistischer Grunderkrankungen.

Abstract

\section{Leukemia cutis in a Patient with Myelogenous Leukemia}

$\nabla$

A 63-year-old man presented with pink to violaceous papules and plaques on the trunk and proximal extremities. His general condition was fine. Histopathology and further hematological investigations led to the diagnosis of leukemia cutis in acute monocytic leukemia of the myelomonocytic subtype.

In about $7 \%$ of patients suffering from leukemia, cutaneous manifestations may be the initial presenting symptom. The pattern of skin lesions varies and may include erythematous macules, papules, nodules, bullae as well as ulcerations. Therefore, the clinical picture of leukemia cutis may have features that overlap with other dermatoses; e.g., drug eruptions, vasculitis, and ulcers of different origin.

\section{Literatur}

1 French-American-British (FAB) Cooperative Group, Bennet JM, Catovsky $D$, Daniel MT. Proposals for the classification of the acute leukaemias. Brit J Haematol 1976; 33: 489-496

2 Vardiman JW, Thiele J, Arber DA et al. The 2008 revision of the World Health Organization (WHO) classification of myeloid neoplasms and acute leukemia: rationale and important changes. Blood 2009; 114 : 37-951

3 Büchner T, Saußele S, Hehlmann R. Akute und chronische myeloische Leukämie. In: Dietel M, Suttorp N, Zeitz M, Hrsg. Harrisons Innere Medizin. Bd 1. Berlin: ABW Wissenschaftsverlag; 2009

4 Pezzutto A, Dörken B. Neoplasien des lymphatischen Systems. In: Dietel M, Suttorp N, Zeitz M, Hrsg. Harrisons Innere Medizin. Bd 1. Berlin: ABW Wissenschaftsverlag; 2009

5 Kresbach H, Kerl H. Myeloproliferative Erkrankungen der Haut. In: Luger A, Gschnait F Hrsg. Dermatologische Onkologie. Wien: Urban u. Schwarzenberg; 1983

6 Kaudewitz P. Leukämien. In: Braun-Falco O, Plewig G, Wolff HH et al., Hrsg. Dermatologie und Venerologie. Heidelberg: Springer; 2005

7 Cerroni L, Zenahlik P, Kerl H. Specific cutaneous infiltrates of B-cell chronic lymphotic leukemia arising at the site of herpes zoster and herpes simplex scars. Cancer 1995; 76: 26-31

8 Jeong KH, Lew BL, Sim WY. Generalized leukaemia cutis from a small cell variant of T-cel prolymphotic leukaemia presenting with exfoliative dermatitis. Acta Derm Venereol 2009; 89: 509- 512

9 Metzler G, Cerroni L, Schmidt H et al. Leukemic cells within skin lesions of psoriasis in a patient with acute myelogenous leukemia. J Cutan Pathol 1997; 24: $445-448$

10 Smoller BR, Warnke RA. Cutaneous infiltrate of chronic lymphocytic leukemia and relationship to primary cutaneous epithelial neoplasms. J Cutan Pathol 1998; 25: 160 - 164

11 Ziemer M, Bornkessel A, Hahnfeld S et al. 'Spezific' cutaneous infiltrate of B-cell chronic lymphocytic leukemia of the site of a florid herpes simplex infection. J Cutan Pathol 2005; 32: 581 - 584

12 Epstein E, MacEachern K. Dermatologic manifestations of the lymphoblastoma-leukemia group. Arch Intern Med 1937; 60: 867 - 875

13 Matutes E, Brito-Babapulle V, Swansburry J. Clinical and laboratory features of 78 cases of T-prolymphocytic leukemia. Blood 1991; 78: $3269-3274$

14 Baer R, Barcos M, Farrell H et al. Acute myelogenous leukemia with leukemia cutis: Eighteen cases seen between 1969 and 1986. Cancer 1989; 63: $2192-2200$

15 Boggs DR, Wintrobe MM, Cartwright GE. The acute leukemias: Analysis of 322 cases and review of the literature. Medicine (Baltimore) 1962; 41: $163-225$

16 Shaikh BS, Frantz E, Lookingbill DP. Histologically proven leukemia cutis carries a poor prognosis in acute nonlymphotic leukemia. Cutis 1987; 39: $57-60$

17 Straus DJ, Mertelsmann R, Koziner B et al. The acute monocytic leukemias: multidisciplinary studies in 45 patients. Medicine 1980; 59: 409- 425

18 Murphy WG, Fotheringham GH, Busuttil A. Skin lesions in chronic granulocytic leukemia: Treatment of a patient with topical nitrogen mustard. Cancer 1985; 55: 2630-2633

19 Long JC, Mihm MC. Multiple granulocytic tumors of the skin. Cancer 1977; 39: $2004-2016$

20 Wagner G. Leucaemia cutis bei akuter Monozytenleukämie. Akt Dermatol 2004; 30: 300 - 305

21 Horlick HP, Silvers DN, Knobler EH. Acute myelomonocytic leukemia presenting as a benign-appearing cutaneous eruption. Arch Dermatol 1990; 126: $653-656$

22 Hansen RM, Barnett J, Hanson G. Aleukemic leukemia cutis. Arch Dermatol 1986; 122: $812-814$

23 Baden JT, Gammon WR. Leukemia cutis in acute myelomonocytic leukemia. Arch Dermatol 1987; 123: 88 - 90

24 Burg G, Schmoeckel C, Braun-Falco O, Wolff HH. Monocytic leukemia. Arch Dermatol 1978; 114: 418-420

25 Hubler WR, Netherton EW. Cutaneous manifestations of monocytic leukemia. Arch Dermatol 1947; 56: 70-89

26 Schadendorf D, Algermissen B, Hamann K et al. Acute monoblastic leukemia with skin nodules in an adult. J Am Acad Dermatol 1993; 28: $884-888$

27 Dummer R. Hautveränderungen bei Leukämien. In: Garbe C, Dummer R, Kaufmann R, Tilgen W, Hrsg. Dermatologische Onkologie. Berlin: Springer; 1997 
28 Stawiski MA. Skin manifestations of leukemias and lymphomas. Cutis 1978; 21: 814-818

29 Bantel E, Undeutsch W, Adam W. Kutane Infiltrate als Frühsymptome einer Neoplasie des myelomonozytären Zellsystems. Akt Dermatol 1998; 14: 7-11

30 Paydas S, Zorludemir S. Leukaemia cutis and leukaemic vasculitis. Br J Dermatol 2000; 143: 773-779

31 Zax RH, Kulp-Shorten CL, Callen JP. Leukemia cutis presenting as a scrotal ulcer. J Am Acad Dermatol 1989; 21: 410-413

32 Costello MJ, Canizares O, Montague M et al. Cutaneous manifestations of myelogenous leukemia. Arch Dermatol 1955; 71: 605-614
33 Seckin D, Senol A, Gurbuz O et al. Leukemic vasculitis: an unusual manifestation of leukemia cutis. J Am Acad Dermatol 2009; 61: 519-521

34 Cho-Vega JH, Medeiros LJ, Prieto VG et al. Leukemia cutis. Am J Clin Pathol 2008; 129: $130-142$

35 Hattori T, Amano H, Nagai Yet al. Leukemia cutis in a patient with acute monocytic leukemia presenting as unique facial Erythema. J Dermatol 2008; 35: 671-674

36 Wagner G, Diaz C. Morbus Morbihan. Akt Dermatol 2003; 29: 300-303

37 Mischinger-Porzolt A, Schauder S, Schauer A. Chronische Monozytenleukämie. Hautarzt 1986; 37: 230-233

38 Metz J, Wilhelm T, Wilke H. Akute Monozytenleukämie. Hautarzt 1982; 33: $165-167$ 